\title{
Hochminiaturisiertes nicht-invasives Messsystem zur Erfassung von Vitalparametern bei Kleinstlebewesen mit drahtloser RFID-/NFC-Ausleseschnittstelle
}

\author{
Patrick Moser ${ }^{1}$, Elke Mackensen ${ }^{1}$, Fabian Rank ${ }^{2}$ \\ ${ }^{1}$ Hochschule Offenburg; Fakultät Elektrotechnik, Medizintechnik und Informatik; \\ Badstraße 24; 77652 Offenburg \\ ${ }^{2}$ VEGA Grieshaber KG; Am Hohenstein 113; 77761 Schiltach
}

\begin{abstract}
Zusammenfassung
Im Rahmen dieses Beitrags wird ein quasi energieautarkes, nicht-invasives Messsystem für Kleinstlebewesen vorgestellt, das Vitalparameter erfasst und diese in einem FRAM-Speicher bis zum Auslesen abspeichert. Durch eine drahtlose RFID-/NFC-Ausleseschnittstelle kann die erfasste Körpertemperatur und der Puls der letzten Wochen ausgelesen werden. Alle Einstellungen des Messsystems können durch einen geeigneten RFID-Reader für Laptops oder durch Smartphones über die NFC-Schnittstelle geändert werden. Das vollständige Aufladen des nur $3 \mathrm{~g}$ leichten und $15 \mathrm{~mm} \times 25 \mathrm{~mm}$ großen Messsystems erfolgt durch eine selbstgedruckte RFID-Reader-Antenne in Verbindung mit einem RFID-Reader und benötigt hierzu weniger als 21 Stunden. Bei vollständig aufgeladenem Energiespeicher ist ein Betrieb von 47 Tagen möglich. Dies wird durch ein speziell für das Messsystem konzipiertes Lade- und Powermanagement erreicht. Neben der Auswahl von energiesparenden Komponenten für die Hardware und deren bestmöglichen Nutzung, wurde die Software so optimiert, dass das Programm schnell und stromsparend abgearbeitet wird. Die Erweiterbarkeit und Anpassung wird durch das modulare Konzept auch in anderen Bereichen gewährleistet.
\end{abstract}

Keywords: nicht-invasives Messsystem, Vitalparameter, Kleinstlebewesen, RFID, gedruckte Elektronik

\section{Einleitung, Stand der Technik und Problemstellung}

Die Messung von Vitalparameter, insbesondere Puls und Temperatur, an Kleinstlebewesen wie Tieren [1], [2] aber auch von Frühgeborenen [3] ist von großer Bedeutung. Eine Messung von Vitalparametern kann zu unter-schiedlichsten Zwecken notwendig sein, z.B. im Rahmen von pharmazeutischen Untersuchungen an Mäusen/Ratten [1] oder der Untersuchung der Auswirkungen der Klimaveränderungen auf bestimmte Tierarten [4]. Vergleichbare invasive Messsysteme setzen bei subkutaner Implantation ein Mindestgewicht des Kleinstlebewesens von 19,5 g voraus [5].

\section{Problemstellung}

Die Herausforderungen und Anforderungen bei der Entwicklung von Messsystemen zur Erfassung von Vitalparametern an Kleinstlebewesen sind recht vielfältig. Zumeist stehen für das Messsystem ein geringes Gewicht und ein quasi energieautarkes
Arbeiten über einen gewissen Zeitraum neben der eigentlichen messtechnischen Funktionalität im Vordergrund. Hinzu kommt oftmals die Forderung, dass das Messsystem ein drahtloses Auslesen der gemessenen Parameter ermöglichen soll. Sowohl in der Forschung als auch bereits kommerziell erhältliche Systeme verfolgen unterschiedliche Ansätze. Insbesondere bei den kommerziell erhältlichen Systemen handelt es sich zumeist um Systeme, die den Tieren implantiert werden müssen, was auf Grund des operativen Eingriffs für kleine Tieren nicht optimal ist. Ein weiterer Nachteil der in der Literatur beschriebenen Systeme ist, dass die Abmessungen und das Gewicht der Messsysteme nicht für Kleinstlebewesen geeignet sind.

Die Anforderungen an das in diesem Beitrag vorgestellte entwickelte Messsystem sind wie folgt: Das Gewicht des Messsystems soll weniger als $3 \mathrm{~g}$ betragen, die Abmessung kleiner als $15 \mathrm{~mm} \times 25 \mathrm{~mm}$. Es sollen Körpertemperaturen im Bereich von $0^{\circ} \mathrm{C}$ und 
$40^{\circ} \mathrm{C}$ und ein Puls mit 5 bis maximal 600 Schläge pro Minute gemessen werden können. Das System soll mindestens einmal pro Stunde die Temperatur und den Puls erfassen. Die gemessenen Daten sollen mindesten einmal am Tag drahtlos ausgelesen werden können. Die Erfassung von Messwerten über einen längeren Zeitraum ohne den Wechsel/das Aufladen eines Energiespeichers ist wünschenswert.

Im Folgenden wird nun das Systemkonzept und die Funktionalität des hochminiaturisierten nicht-invasives Messsystems zur Erfassung von Puls und Temperatur vorgestellt, das insbesondere für kleine Vögel/Küken im Ruhezustand [4] konzipiert ist. Das Messsystem eignet sich jedoch auch für andere Kleinstlebewesen.

\section{Systemkonzept und Funktionalität}

In Abb. 1 ist das Systemkonzept des nichtinvasiven Messsystems zur Erfassung von Puls und Temperatur bei Kleinstlebewesen dargestellt. Auf Grund der energetischen und platzbedingten Anforderungen wurde als drahtlose Kommunikationsschnittstelle eine aktive Funktechnologie wie Bluetooth ausgeschlossen. Ein vielversprechender Ansatz ist der Einsatz einer RFID- bzw. NFCSchnittstelle zur drahtlosen Kommunikation. In Betracht gezogen wurde hier zunächst ein hochintegrierter SoC (System on Chip) für biomedizinische Anwendungen [6]. Auf Grund seiner recht großen Chipabmessungen (ohne Gehäuse $1,52 \mathrm{~mm} \times 3,2 \mathrm{~mm}$ ) und einer Leistungsaufnahme von ca. $600 \mu \mathrm{W}$ wurde dieser jedoch nicht für diese Anwendung eingesetzt. Nach ersten Designstudien [7] wurde stattdessen ein kommerziell erhältlicher RFID-/NFC-Chip mit Abmessungen von $3 \mathrm{~mm} \times 3 \mathrm{~mm}$ ausgewählt, der zudem auch als ungehäuster Chip verfügbar ist. Neben der drahtlosen Kommunikation ermöglicht der RFID-/NFC-Chip zudem das Harvesting von Energie, sobald der Chip sich in einem entsprechenden magnetischen Feld befindet. Sollte die vom RFID-/NFC-Chip bereitgestellte Spannung zu stark belastet werden und somit unzulässig weit absinken, ist keine Kommunikation mehr möglich. Zur Aufbereitung und Begrenzung der vom RFID/NFC-Chip bereit gestellten Energie wird ein Harvester-Chip eingesetzt. Durch das Festlegen des MPP (Maximum Power Points), wird sichergestellt, dass die $53 \mathrm{kbit} / \mathrm{s}$ schnelle Kommunikation während des Ladens nicht gestört wird, und die maximal zur Verfügung stehende Leistung geerntet werden kann. Bei der Auswahl der sonstigen Komponenten der Messelektronik wurde insbesondere auf eine geringe Leistungsaufnahme im aktiven Betrieb geachtet. Erreicht wird dies beispielsweise durch einen Mikrocontroller mit FRAMSpeicher (Ferroelectric Random Access Memory). Die Pulsmessung basiert auf einem Sensor mit integriertem Infrarotemitter und Photodiode, so dass nach dem Prinzip der Photoplethysmographie der Puls erfasst werden kann. Der zum Einsatz kommende Temperatursensor, mit lediglich zwei elektronischen Anschlüssen, gibt bei Aktivierung entsprechend der Temperatur Strom-Pulse aus. Das Messsystem wird über einen wieder aufladbaren Energiespeicher mit Energie versorgt. Dieser kann durch die Energy-Harvesting-Funktion des RFID-/NFCChips wieder aufgeladen werden, sobald sich das System in einem magnetischen Feld befindet.

Abb. 2 zeigt eine beispielhafte Nutzung des Messsystems an einem Vogelküken. Das Nest des Vogels wird über der RFID-ReaderAntenne positioniert. Wie in [7] beschrieben, bewegt sich das Küken kaum, weshalb der $3 \mathrm{~g}$ leichte Sensor auf dem Rücken des selbst nur $15 \mathrm{~g}$ leichten Vogels positioniert werden kann. Die mit einem flexiblen Leitungsteil abgesetzten Temperatur- und Pulssensoren können an den entsprechenden Positionen des Kleinstlebewesens angebracht werden.

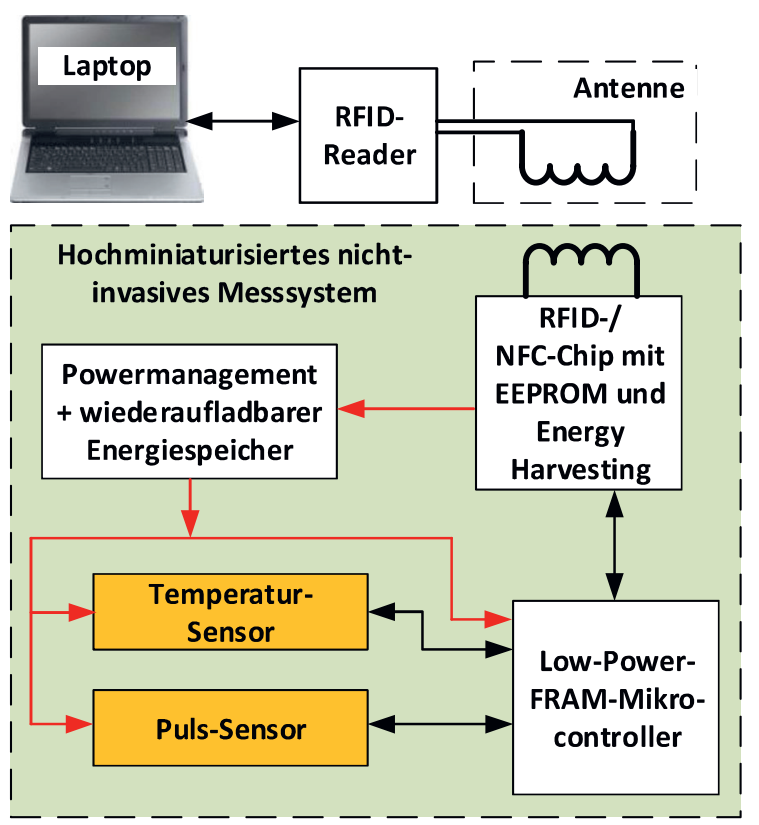

Abb. 1: Blockschaltbild des nicht invasiven Messsystems zur Erfassung von Puls und Temperatur an Kleinstlebewesen 


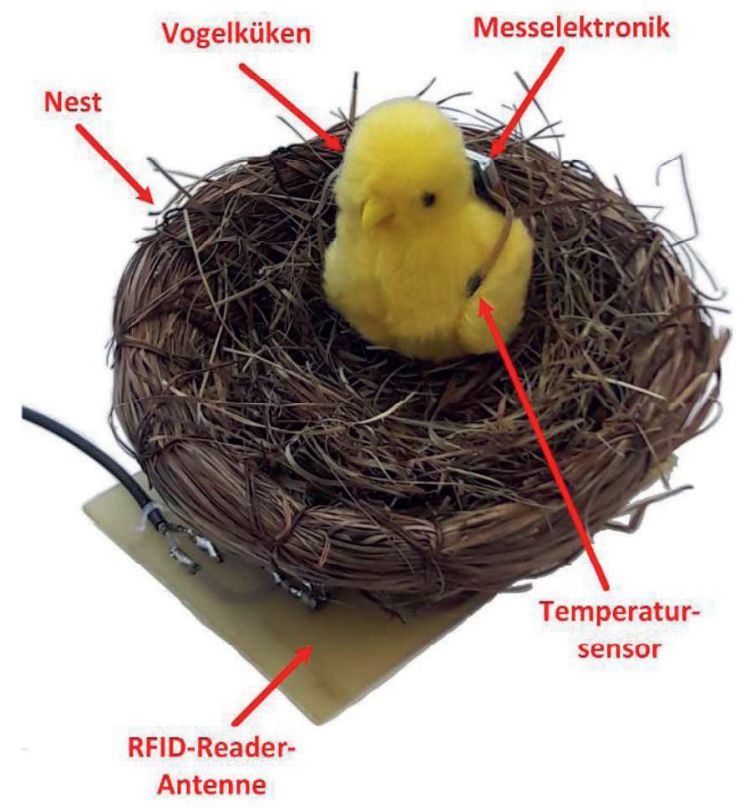

Abb. 2: Beispielhafte Nutzung des nicht invasiven Messsystems bei einem Vogelküken

\section{RFID-Reader mit gedruckter Antenne}

Mit zum Gesamtsystem gehört eine selbst entworfene Antenneneinheit, die unter dem Vogel/Tier platziert ist. Diese Antenneneinheit kann mit einem entsprechenden RFID-/NFCLesegerät über eine SMA-Steckverbindung verbunden werden, um die Messdaten drahtlos per Laptop auszulesen, oder eine Aufladung des Energiespeichers durchzuführen. Alternativ kann in Betracht gezogen werden, die Daten per Smartphone mit der NFCSchnittstelle auszulesen. Als RFID-Reader wurde der "ID ISC.MR102-USB" von Feig Electronic ausgewählt [8], welcher durch eine selbst entworfene grafische Benutzeroberfläche gesteuert wird. Mit dieser Benutzeroberfläche kann das Messsystem konfiguriert werden. Dies betrifft die Auswahl des gewünschten Messintervalls, welche Messgröße gemessen werden soll, sowie verschiedene Einstellungen des Pulssensors.

Eine eigene Entwicklung der Antennen war notwendig, da keine passende, kommerziell erhältliche Antenne nutzbar war. Abb. 3 zeigt die selbstentwickelte Antenne, mit einer Abmessung von $110 \mathrm{~mm}$ x $90 \mathrm{~mm}$. Bei der Antenne handelt es sich um eine mit dem Voltera V-One-Platinendrucker (siehe https://www.voltera.io/) gedruckte Antenne auf $2 \mathrm{~mm}$ dickem FR4-Platinenmaterial. Dies ermöglicht eine schnelle und kostengünstige Herstellung von wenigen Antennen, welche an die Anforderungen angepasst sind. Mithilfe eines Schutzlackes werden die Leiterbahnen und Komponenten vor Umwelteinflüssen, wie Feuchtigkeit geschützt. Durch Verwendung von Folien oder dünnem FR4-Platinenmaterial kann mit dem Platinendrucker auch eine flexible Antenne produziert werden, welche sich optimal an die räumlichen Gegebenheiten anpasst.

Die in Abb. 4 gezeigte Messaufnahme der RFID-Reader-Antenne zeigt deren Impedanzverlauf von $13 \mathrm{MHz}$ bis $14 \mathrm{MHz}$. Der angestrebte Impedanzwert von $50 \mathrm{Ohm}$ bei der Frequenz von $13,56 \mathrm{MHz}$ wurde mit $51 \mathrm{Ohm}$ nicht ganz erreicht. Abb. 5 zeigt die Reflektion der Antenne im Bereich von $13 \mathrm{MHz}$ bis 14 $\mathrm{MHz}$. Je geringer der Wert ist, desto besser überträgt die Antenne die Energie zum Messsystem. Auch hier wurde der beste Wert nicht bei der gewünschten Resonanzfrequenz von $13,56 \mathrm{MHz}$, sondern bei $13,59 \mathrm{MHz}$ erreicht. $\mathrm{Zu}$ beachten ist, dass durch metallische Strukturen, wie es beispielsweise das Messsystem besitzt, die ermittelten Werte der RFID-Reader-Antenne verändert werden. Wird das Messsystem über der RFID-ReaderAntenne positioniert, verschiebt sich die Impedanz und die Resonanz auf die gewünschten Werte, wodurch das System optimal arbeitet. Weitere in der Umgebung vorhandene Messsysteme verändern die Parameter kaum, wodurch theoretisch mehr als ein Messsystem gleichzeitig durch einen RFID-Readern ausgelesen und geladen werden könnten.

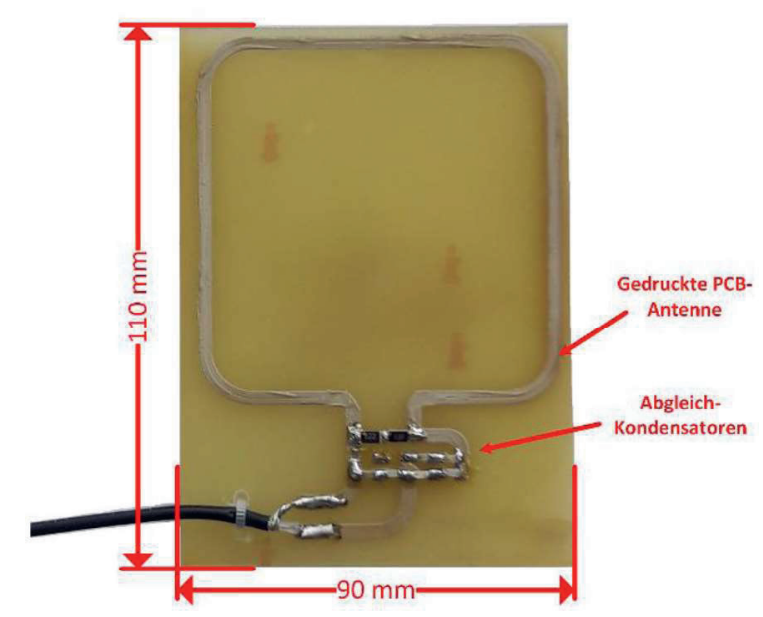

Abb. 3: RFID-Reader mit gedruckter Antenne 


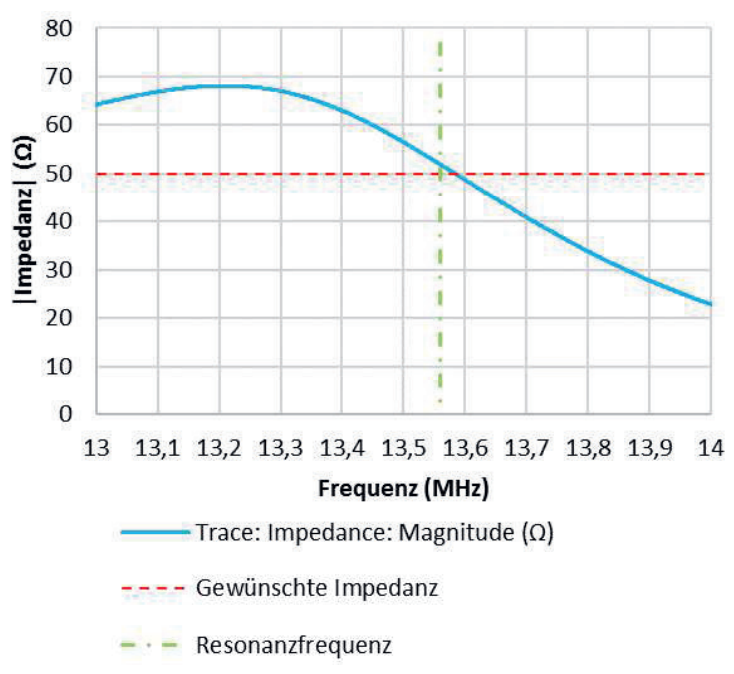

Abb. 4: Impedanz der RFID-Reader-Antenne im Frequenzbereich 13 bis $14 \mathrm{MHz}$

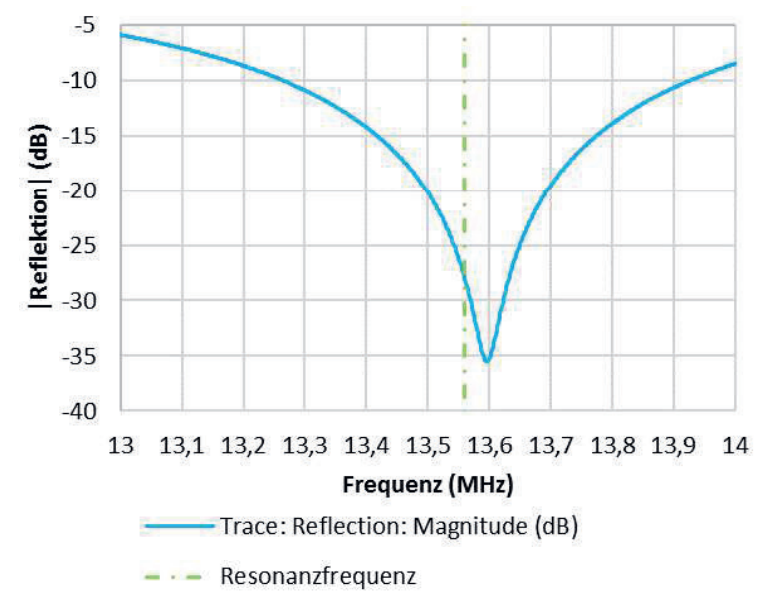

Abb. 5: Reflektion der RFID-Reader-Antenne im Frequenzbereich 13 bis $14 \mathrm{MHz}$

\section{Hardware des nicht-invasiven Messsystems und Powermanagement-Strategien}

Abb. 6 zeigt die Hardware des nicht-invasiven Messsystems, die so konstruiert ist, dass der RFID-/NFC-Chip, der Harvester-Chip mit dem Powermanagement, der Energiespeicher und der Mikrocontroller auf einem 4-lagigen Platinenteil der Abmessung $15 \mathrm{~mm} \times 25 \mathrm{~mm}$ platziert sind. Dieser $1 \mathrm{~mm}$ dicke Platinenteil wird am Beispiel des Vogels auf dessen Rücken platziert (siehe Abb. 2). Aus diesem Platinenteil ragen seitlich flexible Leitungsteile heraus, an dessen Ende sich jeweils der Temperatur- und Pulssensor befinden. Durch die flexiblen Leitungsteile können die Sensoren an die entsprechenden Körperstellen geführt werden, wo die Messwerte erfasst werden müssen. Die Platine enthält für die Datenübertragung per RFID/NFC eine integrierte PCB-Antenne. Durch die Integration in die Platine wird kein externes Bauteil benötigt, wodurch Gewicht und Platz eingespart wird. Die Antenne besteht aus vier Windungen, welche sich auf alle Lagen der Platine aufteilen. Die Elektronik auf der Platine wird durch einen Schutzlack vor Feuchtigkeit geschützt.

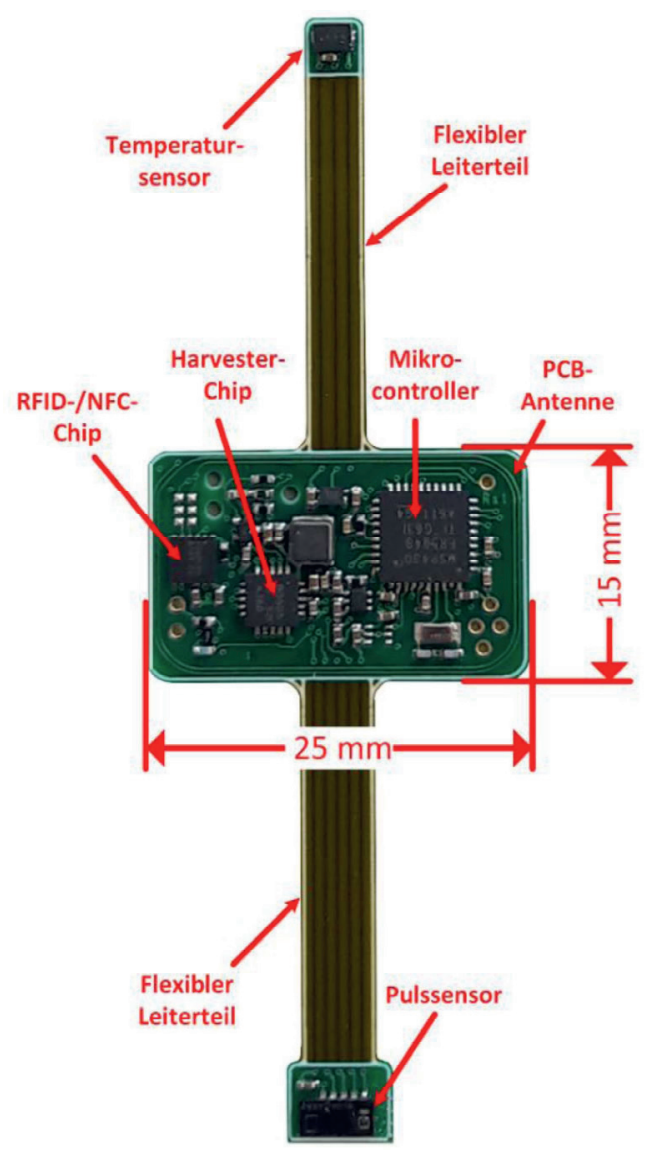

Abb. 6: Aufbau der Messelektronik mit flexiblen Leiteranschlüssen für die Sensoren

Die Hardware und Arbeitsweise des Messsystems sind so konzipiert, dass das Gesamtsystem extrem stromsparend arbeitet. Details hierzu werden im Folgenden beschrieben:

- Durch die Verwendung eines Low-PowerFRAM-Mikrocontrollers, der die überwiegende Zeit im Sleep-Mode arbeitet, kann der Ruhestrom des Systems auf wenige $\mu \mathrm{A}$ reduziert werden.

- Durch das Powermanagement, welches durch den Mikrocontroller gesteuert wird, werden alle nicht benötigten Komponenten von der Spannungsversorgung getrennt. Hierdurch entfallen eventuell auftretende Leckströme, was zu einer weiteren Reduzierung des Ruhestromes und somit auch der durchschnittlichen Stromaufnahme führt. 
- Trotz der am RFID-/NFC-Chip abgeschalteten Versorgungsspannung, wird durch inn am Mikrocontroller ein Interrupt ausgelöst, sobald sich das Messsystem in einem entsprechenden RFID-/NFC-Feld befindet. Anschließend kann der RFID/NFC-Chip durch den Mikrocontroller aktiviert werden, wodurch er seine volle Funktionalität bezüglich Kommunikation und Bereitstellen von Energie erlangt. Dies ermöglicht das sofortige Auslesen der ermittelten Messdaten, die im nichtflüchtigen Speicher des Mikrocontrollers abgelegt werden. Bei einem Messintervall von einer Messung pro Stunde, können für einen Zeitraum von über einer Woche Messwerte gespeichert werden.

- Um das System für den genannten Zeitraum mit Energie zu versorgen, wird die vom RFID-/NFC-Chip bereitgestellte Energie in einen $259 \mathrm{mWh}$ großen LithiumPolymer-Akkumulator gespeichert. Dies geschieht durch einen Harvester-Chip, welcher die benötigte Ladespannung für den Energiespeicher erzeugt und zusätzlich das Laden überwacht.

- Zur Ermittlung der Temperatur des Lebewesens wird die Hauttemperatur gemessen. Hierzu muss der Sensor direkten Kontakt zur Haut besitzen. Der Temperatursensor kommuniziert über Strom-Pulse mit dem Mikrocontroller. Die hierbei entstehenden Analogenwerte werden in einem Komparator mit SchmittTrigger digitalisiert und im Mikrocontroller gezählt. Dieser Vorgang wurde so konzipiert, dass keine Rechenzeit benötigt wird, wodurch Energie eingespart wird.

- Um den Puls des Lebewesens nicht-invasiv zu ermitteln, wird ein Pulssensor eingesetzt, der nach dem Photoplethaysmographie-Verfahren arbeitet. Bei diesem Verfahren leuchtet ein Infrarotemitter auf eine Arterie. Durch die sich periodisch ändernde Absorptionsrate, welche bei einem Herzzyklus entsteht, kann auf den Puls rückgeschlossen werden. Der Pulssensor liefert bereits digitale Werte, welche im Mikrocontroller zwischen der Abtastrate des Sensors verarbeitet werden. Hierdurch entfällt die Notwendigkeit eines großen Speichers im Mikrocontroller, um die ermittelten Daten zwischen zu speichern. Um eine präzise Aussage über den Puls treffen zu können, wird dieser eine Minute überwacht. Der Sensor kann für die jeweiligen physikalischen Gegebenheiten, wie Dicke der Haut oder des Gewebes, sowie die zu erwartenden Herzfrequenz des Lebewesens angepasst werden. Hierzu lassen sich die Anzahl der Messungen pro Sekunde, Leuchtintensität und Modulationsfrequenz des integrierten Infrarotemitters einstellen.

- Die durch die Messung berechneten Vitalparameter werden im FRAM-Speicher des Mikrocontrollers abgelegt, bis diese dann per RFID/NFC ausgelesen werden.

\section{Energetische Betrachtung des Messsystems}

In Abb. 7 ist die Leistungsaufnahme des Messsystems für eine Messung des Pulses und der Temperatur in einem Messintervall von einer Stunde nicht maßstabsgetreu grafisch dargestellt. Das Messsystem hat bei aktivem Betrieb und der geforderten Messdatenerfassung einmal pro Stunde, eine durchschnittliche Leistungsaufnahme von ca. $115 \mu \mathrm{W}$. Mit dem zum Einsatz kommenden hochminiaturisierten, wieder aufladbarem Energiespeicher, der über eine Kapazität von $259 \mathrm{mWh}$ verfügt, und dem man ca. $50 \%$ seiner Energie bis zur Wiederaufladung entnehmen kann, kann das System ca. 47 Tage die Datenerfassung ohne Notwendigkeit der Energieaufladung erfolgen. Dies ist möglich, da sich das System die meiste Zeit im Sleep-Mode befindet. In diesem Zustand hat das Messsystem lediglich eine Leistungsaufnahme von $37 \mu \mathrm{W}$. Für Berechnungen und Initialisierungen arbeitet der Mikrocontroller mit einem Takt von $8 \mathrm{MHz}$. Hierbei wird für eine kurze Zeit vor und nach den Messungen eine Leistung von $3256 \mu \mathrm{W}$ benötigt. Für die Messung der Temperatur sind es $370 \mu \mathrm{W}$, was die Kommunikation des Temperatursensors mit dem Mikrocontroller bereits beinhaltet. Der Mikrocontroller selbst benötigt während der Kommunikation kaum Energie, da er sich im Sleep-Mode befindet. Der Pulssensor benötigt bei sieben Messungen pro Sekunde, wobei der Infrarotemitter für kurze Zeit eine Stromaufnahme von $100 \mathrm{~mA}$ besitzt, was einer Leistungsaufnahme von $1184 \mu \mathrm{W}$ entspricht. Bei dieser Messung verarbeitet der Mikrocontroller die erhaltenen Messwerte sofort, was zu einer gesamten Leistungsaufnahme von $4440 \mu \mathrm{W}$ führt. Wird die Zeit zwischen den Temperatur- und Pulsmessungen vergrößert, sinkt die durchschnittliche Leistungsaufnahme, wodurch auch eine deutlich größere Betriebsdauer erreicht wird. 


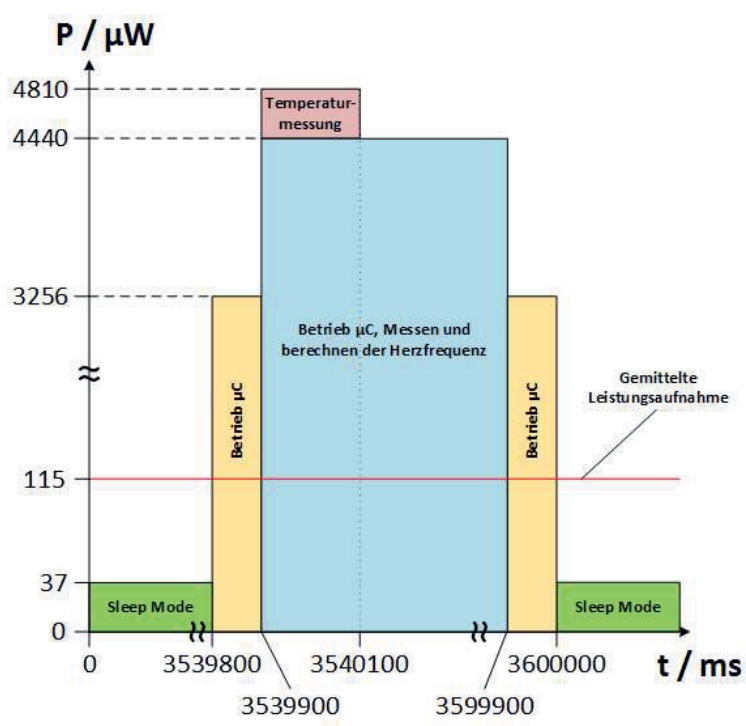

Abb. 7: Leistungsaufnahme des Messsystems für eine Messung des Pulses und der Temperatur in einem Messintervall von einer Stunde

Der Energiespeicher der Messelektronik wird durch das von der RFID-Reader-Antenne erzeugte magnetische Feld aufgeladen, da der im Messsystem eingesetzte RFID-/NFC-Chip das Energy-Harvesting der Energie aus dem magnetischen Feld ermöglicht. In Abb. 8 ist aufgezeigt, welche Leistung aus dem Magnetfeld in Abhängigkeit der Ausgangsspannung am RFID-/NFC-Chip für unterschiedliche Abstände zwischen der RFIDReader-Antenne und der PCB-Messsystemantenne geerntet werden kann. Die PCBEmpfangsantenne ist parallel und mittig über der selbstgedruckten RFID-Reader-Antenne ausgerichtet. $\mathrm{Zu}$ beachten ist, dass bei steigendem Abstand die mögliche Leistung, mit der der Energiespeicher geladen werden kann, abnimmt. Ab einem Abstand der Antennen von mehr als $100 \mathrm{~mm}$ ist das Laden, sowie die Kommunikation nicht mehr möglich. Die vom RFID-Reader bereitgestellte Leistung kann nicht eingestellt werden und beträgt laut Datenblatt [8] maximal 1,2 W. Die Leistung ist nur dann maximal, wenn die beiden Antennen parallel zueinander ausgerichtet sind. Mit steigendem Winkel reduziert sich die übertragene Leistung. Neben metallischen Materialen können auch weitere Materialien einen Einfluss auf die Leistung haben.

Zur optimalen Leistungsentnahme aus dem RFID-Feld wird der MPP des Harvester-Chips auf $65 \%$ der möglichen Leerlaufspannung gesetzt. Dies entspricht circa 2,3 V, was bei den beiden in Abb. 8 gezeigten Abstände der Antenne dem Leistungsmaximum entspricht. Hierdurch kann unabhängig der Abstände der
$259 \mathrm{mWh}$ große Energiespeicher schnellstmöglich geladen werden. Bei einem Abstand der Antennen von $30 \mathrm{~mm}$ entspricht dies $14800 \mu \mathrm{W}$. Wird von einem Wirkungsgrad des Harvester-Chips von $85 \%$ ausgegangen, kann der Energiespeicher in unter 21 Stunden komplett geladen werden. Dies sollte vor dem Anbringen an das Kleinstlebewesen geschehen. Der Energiespeicher kann beim Auslesen der Messwerte nachgeladen werden. Bei einem täglichen Nachladen reicht - bei optimaler Ausrichtung der Antennen zueinander - eine Zeit von weniger als 15 Minuten.

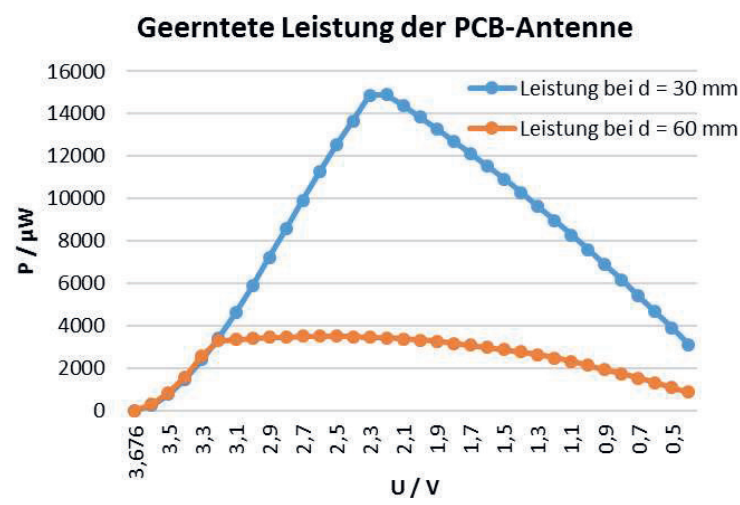

Abb. 8: Geerntete Leistung an der PCBEmpfangsantenne des Messsystems bei unterschiedlichen Abständen in der Mitte der selbstgedruckten RFIDReader-Antenne

\section{Zusammenfassung und Ausblick}

Das in diesem Beitrag vorgestellte nichtinvasive Messsystem ermöglicht es, die Vitalparameter von Kleinstlebewesen, wie z.B. Vogelküken für einen gewissen Zeitraum energieautark zu überwachen. Die wichtigsten Kenndaten des Messsystems sind im Folgenden zusammenfassend aufgeführt:

- Mit dem Messsystem lassen sich Körpertemperaturen im Bereich von $0^{\circ} \mathrm{C}$ bis $40^{\circ} \mathrm{C}$ überwachen.

- Die optische Ermittlung des Pulses nach dem Photoplethaysmographie-Verfahren ermöglicht eine Pulsmessung von 5 bis maximal 600 Schläge pro Minute.

- Die Hauptplatine des Messsystems besitzt eine Abmessungen $15 \mathrm{~mm} \times 25 \mathrm{~mm}$ und einen über RFID/NFC wieder aufladbaren Energiespeicher. Per RFID/NFC können die gemessenen Vitalparameter drahtlos mit $53 \mathrm{Kbit} / \mathrm{s}$ ausgelesen werden. Dies kann über einen speziellen RFID-Reader oder ein Smartphone mit NFC-Schnittstelle geschehen. 
- Die genutzten Sensoren sind durch flexible Leitungsteilen von der Hauptplatine abgesetzt, um diese an die entsprechenden Körperstellen positionieren zu können.

- Das System ist momentan dafür ausgelegt mindestens einmal pro Stunde die Temperatur und den Puls zu erfassen. Für das Messintervall von einer Stunde und mit dem gewählten miniaturisierten Energiespeicher beträgt die Leistungsaufnahme des Messsystems $115 \mu \mathrm{W}$, wodurch ein energieautarkes Arbeiten von 47 Tagen erreicht wird.

- Bei täglichem Laden des Energiespeichers von nur 15 Minuten über das Magnetfeld eines RFID-Readers, kann das Messsystem 24 Stunden energieautark betrieben werden.

- Das Messsystem, welchen am Kleinstlebewesen angebracht wird, wiegt lediglich $3 \mathrm{~g}$.

Das hier vorgestellte Messsystem kann prinzipiell auch für andere Anwendungsbereiche eingesetzt und angepasst werden. Neben der Überwachung von Kleinstlebewesen wie Tieren, könnte z.B. durch leichte Anpassung der Messelektronik ein Wearable zur Überwachung von Säuglingen entstehen. Durch den modularen und somit flexiblen Aufbau der Messelektronik könnte zudem das System durch andere Sensoren ausgestattet werden, wodurch andere Messgrößen erfasst werden könnten. Beispielsweise denkbar wäre die Überwachung von Temperatur und Luftfeuchtigkeit beim Transport von Waren, um eine unterbrochene Kühlkette oder falsche Lagerung von Waren zu erkennen.

\section{Literaturnachweis}

[1] Volk, T.: Semipassives Sensorimplantat mit RFID ähnlicher Schnittstelle zur Überwachung der Vitalfunktionen von Kleintieren.

Dissertation, Hochschule Offenburg \& Universität Freiburg, 2014

[2] McCafferty, D.J; Gallon, S.; Nord, A. (2015): Challenges of measuring body temperatures of free-ranging birds and mammals. In. Animal Biotelemetry (2015). pages 1-10. DOI 10.1186/s40317-015-0075-2

[3] Flügel, M.: Kontinuierliche Messung der Körperkerntemperatur bei Frühgeborenen mittels eines nicht-invasiven Sensors und Korrelation mit der durch Nah-InfrarotSpektroskopie gemessenen regionalen zerebralen Sauerstoffsättigung. Dissertation, Universität Lübeck, 2013.

[4] Kuepper, N.D.; et. al.: Facultative hypothermia as a survival strategy during snowstorm induced food shortages in Antarctic stormpetrel chicks. In: Comparative Biochemistry and Physiology Part A. Molecular \& Integrative
Physiology, Volume 224, October 2018, Pages 76-83; DOI 10.1016/j.cbpa.2018.06.018

[5] DSI: Guide to DSI's - Telemetry Devices, Extra-Small Implants, 2019

[6] Bhattacharyya, M.; Dusch, B.; Jansen, D.; Mackensen, E.: Design and Verification of a Mixed-Signal SoC for Biomedical Applications. In: Proceeding of the 54. MPC-Workshop. UIm, Juli 2015. Pages 43-48. ISSN 1868-9221

[7] Volk, F.: Entwicklung einer drahtlosen hochminiaturisierten Elektronik zur Untersuchung von antarktischen Sturmschwalbenküken. Masterthesis, Hochschule Offenburg, 2016.

[8] Feig Electronic: HF Mid Range Reader ID ISC.MR102, Datasheet, Dezember 2017 